\title{
Numerical Simulation of Maxwell's Equations
}

\author{
Mai Mismar \\ Al-Quds Open UniversityNablus-Palestine \\ mmismar@qou.edu
}

\begin{abstract}
In this paper, we propose the finite-difference time-domain (FDTD) scheme based on the Yee algorithm to solve the time dependent Maxwell's equations. Where the volume domain has been discretized with a regular, structured, staggered and rectangular grid. This method transforms Laplace and Poisson equations into an equivalent matrix form that can be solved by a suitable numerical method. Then we use the Gauge transformation condition to convert both the electric and magnetic fields to scalar and vector potentials respectively. This can be described by Laplace and Poisson equations. These equations can be solved by the well known finite difference method. To show the efficiency of this method we consider a structure of a simple $5 \times 5$ grid of voltage samples.
\end{abstract}

Keywords: Maxwell's equations, Yee algorithm, FDTD

\section{INTRODUCTION}

Maxwell's equations are one of the most important models in different fields. They describe electromagnetic phenomena such as micro, radios and radar waves. The modeling of systems involving electromagnetic waves is widely spread and has attracted the attention of many authors and researchers. The set of basic equations which Maxwell constructed become known as the Maxwell's equations and are given in their differential formulation $[8,9,17,18,19]$ :

Farady's Law:

$$
\frac{\partial \vec{B}}{\partial t}=-\nabla \times \vec{E}-\vec{M}
$$

Amper's Law:

$$
\frac{\partial \vec{D}}{\partial t}=-\nabla \times \vec{H}-\vec{J}
$$

Gauss's Law:

$$
\begin{aligned}
& \nabla \cdot \vec{D}=\rho \\
& \nabla \cdot \vec{B}=\rho^{*}
\end{aligned}
$$

Continuity Equation:

$$
\begin{aligned}
\nabla \cdot \vec{J} & =-\frac{\partial}{\partial t} \rho \\
\nabla \cdot \vec{M} & =-\frac{\partial}{\partial t} \rho *
\end{aligned}
$$


where $B$ is the magnetic flux density, $D$ is the electric flux density, $E$ is the electric field intensity, $H$ is the magnetic field intensity, $J$ is the electric current density, $M$ is the magnetic current density, $\rho$ is the electric charge density and $\rho^{*}$ is the magnetic charge density. The flux densities and the field densities are related through the constitutive relations. For linear and isotropic media, these are:

$$
\begin{aligned}
& D=\varepsilon E=\varepsilon_{0} \varepsilon_{r} E \\
& B=\mu H=\mu_{0} \mu_{r} H
\end{aligned}
$$

where $\varepsilon_{0}$ is the free space permittivity $\left(8.854 \times 10^{-12} F / m\right), \varepsilon_{r}$ is the relative permittivity and $\varepsilon$ is the permittivity of the media. Likewise, $\mu_{0}$ is the free space permeability $\left(4 \pi \times 10^{-7} H / m\right), \mu_{r}$ is the relative permeability and $\mu$ is the permeability of the media. The variety of applications based on electromagnetism is enormous. Unfortunately, in the case where a solution of the Maxwell equations is required, it is not possible to solve them analytically. It can be solved analytically only for a simple domains such as a sphere and an infinite circular cylinder $[13,19,22]$. Nowadays the modeling of systems involving electromagnetic waves is widely done through the solution of the time domain Maxwell equations on space grid. Such systems were solved by many different methods. The first method is the Finite Difference Time Domain Method ( FDTDM ), that proposed by K.Yee $[19,22]$. This method is very well known for its simplicity and efficiency, but it is difficult to generalize to unstructured non-Cartesian grids and suffer from the inaccurate representation of the solution on curved boundaries $[14,19,22]$. Another method that has been developed and based on unstructured grids and can deal with complex geometries is the Finite Element Time Domain Method (FETDM ) [4, 16]. However, some of major difficulties appear when using the standard Finite Element Method ( FEM ) is that its use on a globally conforming mesh, that means, a mesh without hanging ( connecting ) nodes or mismatch of mesh points along internal boundaries. Moreover, the difficulty in representing corner singularities [4, 16]. The Discontinuous Galerkin Methods ( DGM ) [3, 6, 7, 10, 12, 14, 23] are mainly based on discontinuous finite element spaces. This can easily handle elements of various types and shapes, irregular non-conforming meshes and even locally varying polynomial degree. Moreover, continuity is weakly enforced across mesh interfaces by adding bilinear forms [14]. The Variational Iteration Method ( VIM ), [1, 2 ,5,11, 14, 20, 21] is proposed by J. He provides a sequence of functions which converges to the exact solution of the problem $[11,12,15]$. It has been shown that this procedure is a powerful tool for solving various kinds of problems. This technique solves the problem without any need to the discretization of the variables, therefore it is not effected by computation round off errors and one is not forced with necessity of large computer memory and time. In this work, a three dimensional finite difference time domain based on Yee Algorithm is reviewed. And the relation between Maxwell's equations and Potential equations is derived. Then a numerical example of voltage samples is solved using FD method.

\section{NUMERICAL TREATMENT OF MAXWELL'S EQUATIONS}

For the numerical simulation of Maxwell's equations (1.1)-(1.6) we will use the Finite-Difference Time-Domain (FDTD). This method was originally proposed by K.Yee in the seminar paper published in 1966 $[9,19,22]$. Yee proposed a discrete solution to Maxwell's equations based on central difference approximations of the spatial and temporal derivatives of the curl-equations. In fact Yee derived a full three-dimensional formulation, and he validated the method with two-dimensional problems. The basic Yee-algorithm is restricted to a regularly-spaced orthogonal grid. This is not amenable to high-fidelity modeling of very complex geometries.

\subsection{The Yee-Algorithm}

This algorithm is based on considering a uniformly spaced rectangular grid in three-dimensions. Each grid cell has dimensions $\Delta x, \Delta y$, a n d $\Delta z$ along each Cartesian axis. The coordinate of a node of the grid can be expressed in discrete form as: $(x, y, z)_{i, j, k}=(i \Delta x, j \Delta y, k \Delta z)$, where $i, j$, and $k$ are integers. Similarly, the time is uniformly discretized as $t=n \Delta t$. Then an arbitrary function $f(x, y, z, t)$ can be expressed at any node within the discrete space using the notation: 


$$
f(x, y, z, t)=f(i \Delta x, j \Delta y, k \Delta z, n \Delta t)=f_{i, j, k}^{n} .
$$

Within this uniform grid, the projections of the vector electric field parallel to a grid edges are sampled at edge grid edge center. Dual to this, the projection of the magnetic field normal to each grid cell face is sampled at the center of a grid face. This is illustrated in figure (2.1). Observing fig. (2.1), it is apparent that the tangential electric field projected on the edges bounding a cell face circulate about the normal magnetic field vectors. This provides the essential pieces to formulate a curl operation. To illustrate this, consider the $\mathrm{x}$-projection of Faraday's law (1.1):

$$
\mu \frac{\partial H_{x}}{\partial t}=\frac{\partial E_{y}}{\partial z}-\frac{\partial E_{z}}{\partial y}-M_{x}
$$

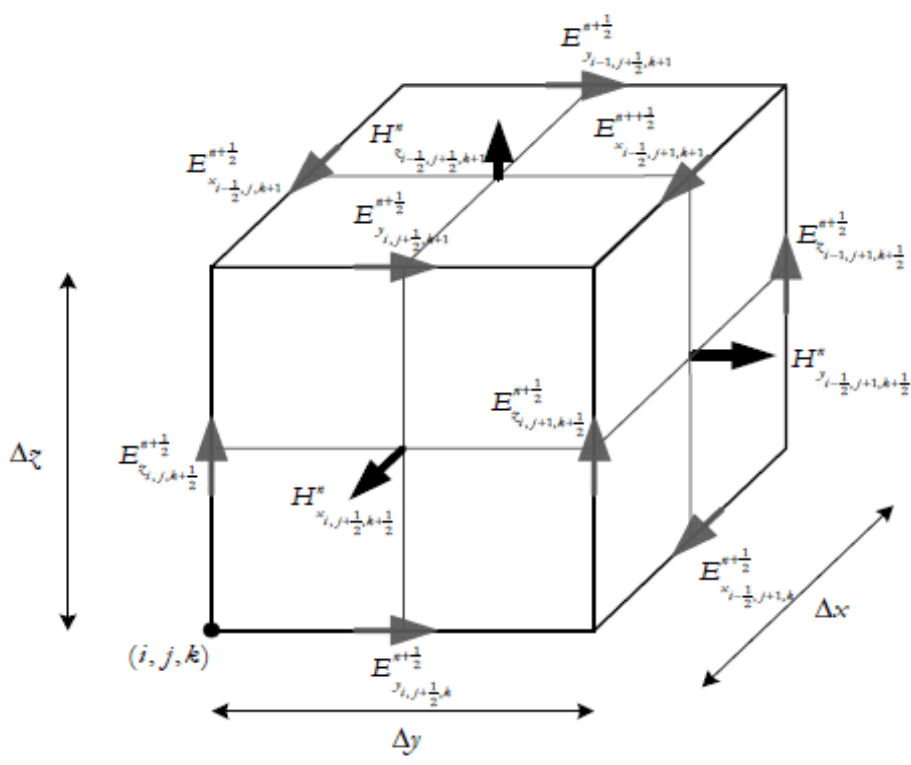

Figure (2.1): Primary grid cell of the regular, structured, rectangular, staggered grip.

where a linear isotropic material is assumed. Using the discretization shown in Fig. (2.1), the time derivative and the spatial derivatives from the curl operator are approximated via central differences, leading to a discrete form of Faraday's law:

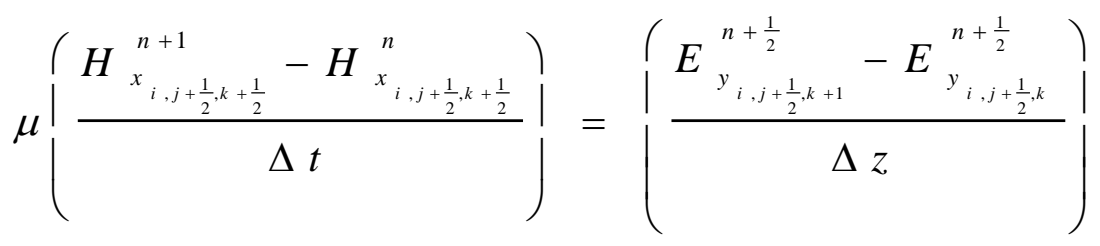

$$
\begin{aligned}
& -\left(\frac{E_{z_{i, j+1, k+\frac{1}{2}}^{n+\frac{1}{2}}-E_{z_{i, j, k+\frac{1}{2}}}^{n+\frac{1}{2}}}^{\Delta y}}{\Delta y}-M_{x_{i, j+\frac{1}{2}, k+\frac{1}{2}}^{n+\frac{1}{2}}} .\right.
\end{aligned}
$$

It is observed from (2.3) that the central difference approximations of both spatial derivatives of the electric field projections are second-order accurate at the face center, which is the sample location of the normal magnetic field. By staggering the magnetic field and the electric field in time, the time-derivative is also secondorder accurate. Consequently, the difference operator in (2.3) is second-order accurate in both space and time. Following the same procedure, one can derive similar expressions for the $y$ and $z$-projections of Faraday's law as well: 


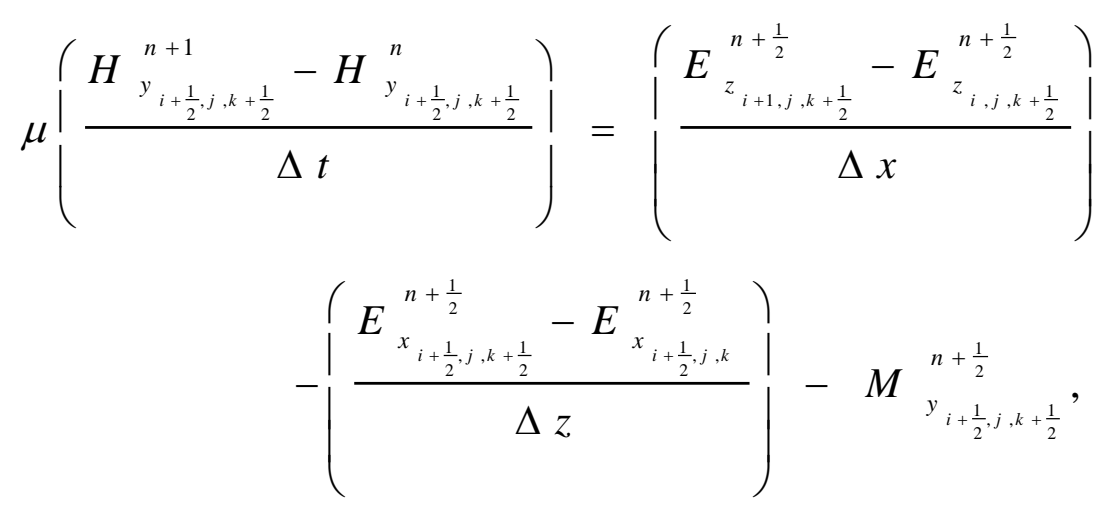

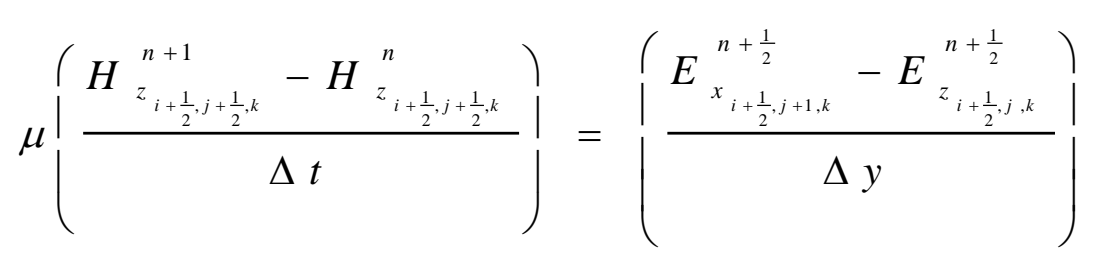

$$
\begin{aligned}
& -\left|\frac{E_{y_{i+1, j+\frac{1}{2}, k}^{n+\frac{1}{2}}-E_{y_{i, j+\frac{1}{2}, k}}^{n+\frac{1}{2}}}^{\Delta x}}{\Delta x}\right|-M_{z_{i+\frac{1}{2}, j+\frac{1}{2}, k}^{n+\frac{1}{2}}}^{n}
\end{aligned}
$$

The discrete form of Ampère's law is derived via a secondary grid cell, as illustrated in Figure (2.2). The secondary grid cell edges connect the cell centers of the primary grid cells illustrated in Fig. (2.1). The secondary grid cell also has dimensions $\Delta x, \Delta y$, a $\mathrm{n} \mathrm{d} \Delta z$. Thus, the edges of the secondary grid pass through the centers of the faces of the secondary grid cells. Dually, the edges of the primary grid

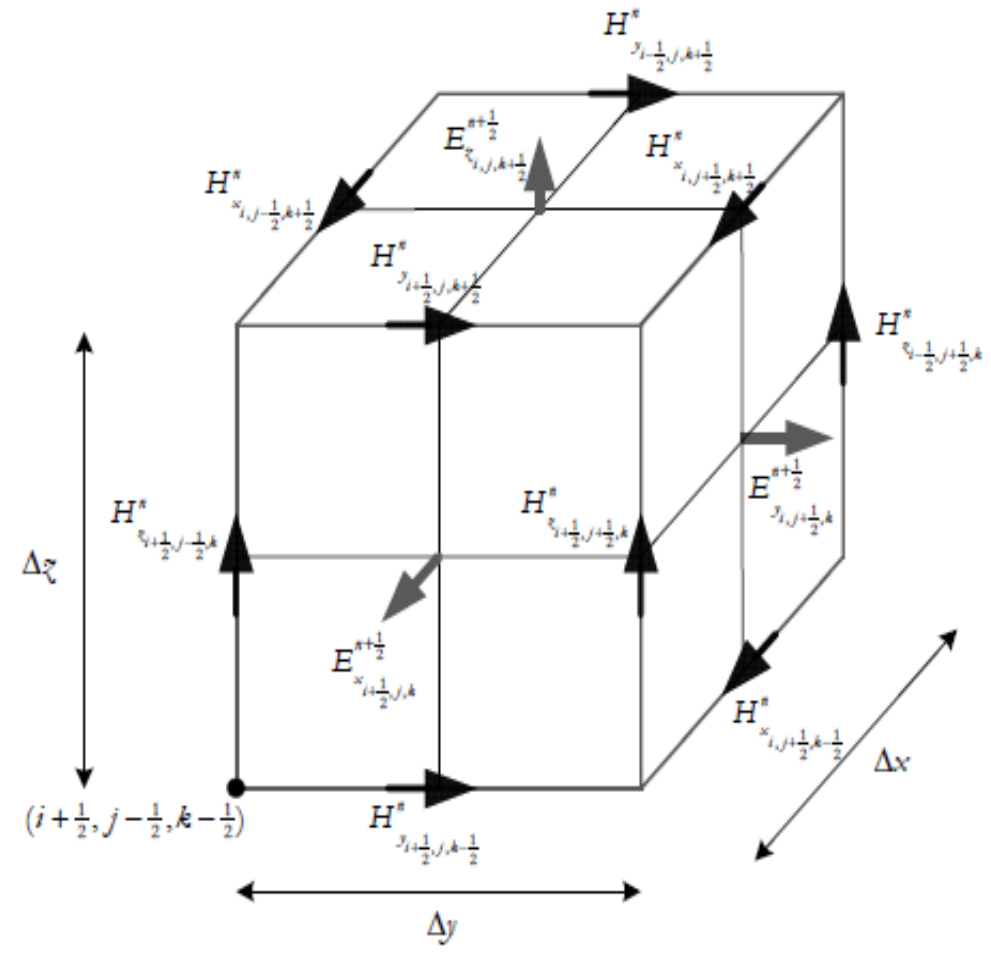

Figure (2.2): Secondary grid cell of the regular, structured, rectangular, staggered grip. 
pass through the face centers of the secondary grid cells. Consequently, the electric and magnetic field vectors have dual roles in the primary and secondary grids. Observing a secondary grid face in Fig. (2.2), it is apparent that the magnetic field lines on a cell face circulates about the normal electric field line. Again, this provides the essential components of a curl operation. The $x$-projection of Ampère's law is expressed in a discrete form as:

$$
\begin{gathered}
\left.\varepsilon\left|\frac{E_{x_{i+\frac{1}{2}, j, k}}^{n+1}-E_{x_{i+\frac{1}{2}, j, k}}^{n-\frac{1}{2}}}{\Delta t}\right|=\mid \frac{H_{z_{i+\frac{1}{2}, j+\frac{1}{2}, k}}^{n}-H_{z_{i+\frac{1}{2}, j-\frac{1}{2}, k}}^{n}}{\Delta y}\right) \\
\left.-\mid \frac{H_{y_{i+\frac{1}{2}, j, k+\frac{1}{2}}^{n}-H_{y i+\frac{1}{2}, j, k-\frac{1}{2}}^{n}}^{n} \mid-J_{x_{i+\frac{1}{2}, j, k}^{n}}^{n}}{\Delta z}\right)
\end{gathered}
$$

where an isotropic, linear, lossless media has been assumed. Similar expressions can be derived for the $y$ and z-projections of Ampère's law. The discrete form of Faraday's and Ampère's laws lead to a total of six equations, which can then be used to solve for the time-dependent vector field intensities. Yee proposed to do this with an explicit time-marching scheme. To this end, it is assumed that the initial values of the discrete fields are known over all space. Subsequently, a recursive solution scheme can be used to advance the fields through time. For example, from (2.3), assuming that $E_{y}^{n+1}, E_{z}^{n+1}$ and $H_{x}^{n}$ are known at all spatial samples, an explicit update operator used to solve for $H_{x}^{n+1}$ is expressed as:

$$
\begin{aligned}
& H_{x_{i, j+\frac{1}{2}, k+\frac{1}{2}}^{n+1}}^{n+}=H_{x_{i, j+\frac{1}{2}, k+\frac{1}{2}}^{n}}^{n}+\frac{\Delta t}{\mu} \mid\left(\frac{E_{y_{i, j+\frac{1}{2}, k+1}}^{n+\frac{1}{2}}-E_{y_{i, j+\frac{1}{2}, k}}^{n+\frac{1}{2}}}{\Delta z}\right)
\end{aligned}
$$

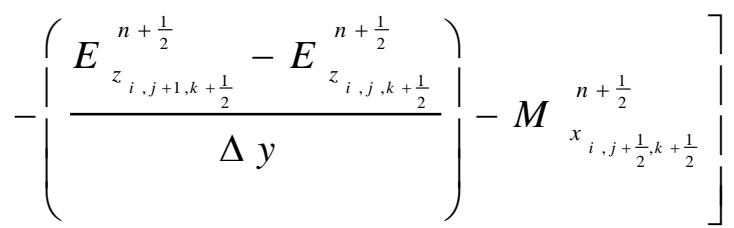

Similarly, we obtain

$$
\begin{aligned}
& H H_{y_{i+\frac{1}{2}, j, k+\frac{1}{2}}}^{n+1}=H \sum_{y_{i+\frac{1}{2}, j, k+\frac{1}{2}}}^{n}+\frac{\Delta t}{\mu} \mid\left(\frac{E_{z_{i+1, j, k+\frac{1}{2}}}^{n+\frac{1}{2}}-E_{z_{i, j, k+\frac{1}{2}}}^{n+\frac{1}{2}}}{\Delta x}\right)
\end{aligned}
$$

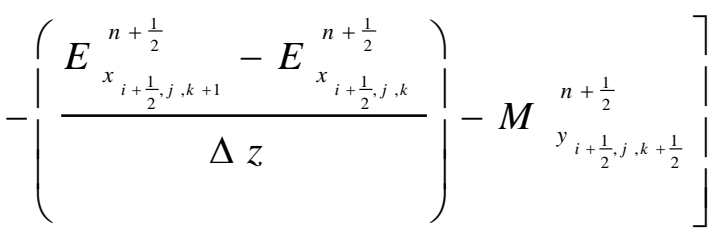




$$
\begin{aligned}
& \left.H_{z_{i+\frac{1}{2}, j+\frac{1}{2}, k}}^{n+1}=H_{z_{i+\frac{1}{2}, j+\frac{1}{2}, k}^{n}}^{n}+\frac{\Delta t}{\mu}|| \frac{E_{x_{i+\frac{1}{2}, j+1, k}}^{n+\frac{1}{2}}-E_{x_{i+\frac{1}{2}, j, k}}^{n+\frac{1}{2}}}{\Delta y}\right) \\
& -\left\{\frac{E_{y_{i+1, j+\frac{1}{2}, k}^{n+\frac{1}{2}}-E_{y_{i, j+\frac{1}{2}, k}}^{n+\frac{1}{2}}}^{\Delta x}}{\Delta x}\left|-M_{z_{i+\frac{1}{2}, j+\frac{1}{2}, k}^{n+\frac{1}{2}}}\right|\right.
\end{aligned}
$$

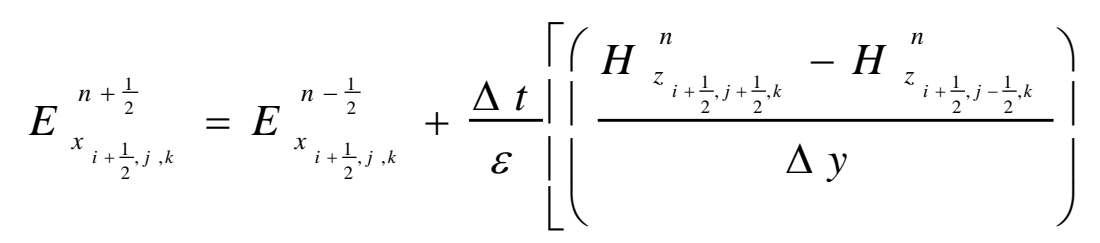

$$
\begin{aligned}
& -\left|\frac{H_{y_{i+\frac{1}{2}, j, k+\frac{1}{2}}^{n}}^{n}-H_{y_{i+\frac{1}{2}, j, k-\frac{1}{2}}}^{n}}{\Delta z}\right|-J_{x_{i+\frac{1}{2}, j, k}^{n}}^{n} \mid \\
& E_{y_{i, j+\frac{1}{2}, k}}^{n+\frac{1}{2}}=E_{y_{i, j+\frac{1}{2}, k}}^{n-\frac{1}{2}}+\frac{\Delta t}{\varepsilon}||\left(\frac{\left.H_{x_{i, j+\frac{1}{2}, k+\frac{1}{2}}^{n}-H_{x_{i, j+\frac{1}{2}, k-\frac{1}{2}}}^{n}}^{\Delta z}\right)}{\Delta z}\right)
\end{aligned}
$$

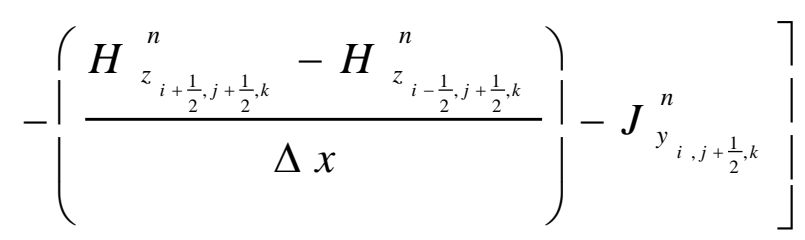

$$
\begin{aligned}
& E_{z_{i, j, k+\frac{1}{2}}}^{n+\frac{1}{2}}=E_{z_{i, j, k+\frac{1}{2}}}^{n-\frac{1}{2}}+\frac{\Delta t}{\varepsilon}||\left(\frac{\left.H_{y_{i+\frac{1}{2}, j, k+\frac{1}{2}}^{n}-H_{y_{i-\frac{1}{2}, j, k+\frac{1}{2}}}^{n}}^{\Delta x}\right)}{\Delta x}\right) \\
& -\left\{\frac{H_{x_{i, j+\frac{1}{2}, k+\frac{1}{2}}^{n}-H_{x_{i, j-\frac{1}{2}, k+\frac{1}{2}}}^{n}}^{\Delta y}\left|-J_{z_{i, j, k}+\frac{1}{2}}^{n}\right|}{\Delta y}\right. \text {. }
\end{aligned}
$$

Equations (2.7)-(2.12) are the first-order difference equations defining Yee's algorithm and are the foundation of the FDTD method $[9,17,19,22]$. These equations provide an explicit recursive update scheme of the electromagnetic fields in linear, isotropic, lossless media throughout the entire volume. 


\subsection{Transforming Maxwell's Equations to Scalar and Vector Potential Equations Using Gauge} Transformation

Electric and magnetic fields can be written in terms of scalar and vector potentials as follows respectively [9, 19]:

$$
\nabla \square E=-\rho / \varepsilon
$$

and

$$
E=-\nabla V
$$

Therefore:

$$
\nabla^{2} V=-\frac{\rho}{\varepsilon}
$$

This is Poisson's equation. At a point in space where the charge density is zero, it becomes

which is generally known as Laplace's equation.

$$
\nabla^{2} V=0
$$

Unlike the electrostatic field, magnetic fields have no sources or charges, and the magnetic lines of force are closed curves. Consequently the surface integral of the magnetic field over a closed surface is zero, and therefore

$$
\nabla \square \mathbf{B}=0
$$

but

$$
\mathrm{B}=\nabla \times \mathrm{A}
$$

and

$$
\begin{gathered}
\nabla \square \mathrm{A}=0 \\
\nabla \times(\nabla \times \mathrm{A})=\mu \mathrm{J} \\
\text { but }(\text { curl curl }=\text { grad div }- \text { nebla squared })
\end{gathered}
$$

using equation (2.21) we arrive with

$$
\nabla^{2} \mathrm{~A}=-\mu \mathrm{J}
$$

This is known as Poisson's equation for the magnetic vector potential.

\section{3- Laplace's and Poisson's Equations in Two Dimensions}

A useful approach to the calculation of electric potential is to relate that potential to the charge density $[9,19,22]$ which gives rise to it. The electric field is related to the charge density by the divergence relationship in equation (2.13), and the electric field is related to the electric potential by a gradient relationship in equation (2.14). Therefore the potential is related to the charge density by Poisson's equation in equation (2.15). In a charge-free region of space, this reduces to Laplace's equation in equation (2.16). Since the potential is a scalar function, this approach has advantages over trying to calculate the electric field directly. Once the potential has beencalculated, the electric field can be computed by taking the gradient of the potential. Laplace's equation in two dimensions may be expressed as

$$
\nabla^{2} \Phi=\frac{\partial^{2} \Phi}{\partial x^{2}}+\frac{\partial^{2} \Phi}{\partial y^{2}}=0
$$

and Poisson's equation in two dimensions may be expressed as:

$$
\nabla^{2} \Phi=\frac{\partial^{2} \Phi}{\partial x^{2}}+\frac{\partial^{2} \Phi}{\partial y^{2}}=F(x, y)
$$

\subsection{Solving Maxwell's Equations using Finite difference Method (FDM)}

The finite difference techniques are based upon approximations which permit replacing differential equations by finite difference equations $[9,19,22]$. These finite difference approximation relate the value of the dependent variable at a point in the solution region to the values at some neighboring points. Constructing finite difference approximations from a given differential equation involves estimating derivatives numerically. The FDTD method employs finite differences as approximations to both the spatial and temporal derivatives that appear in 
Maxwell's equations. Then at any point in the domain D is the average of the values of at the four surrounding points in the 5-point stencil of Figure (3.1). And this corollary is the basis of the iterative method

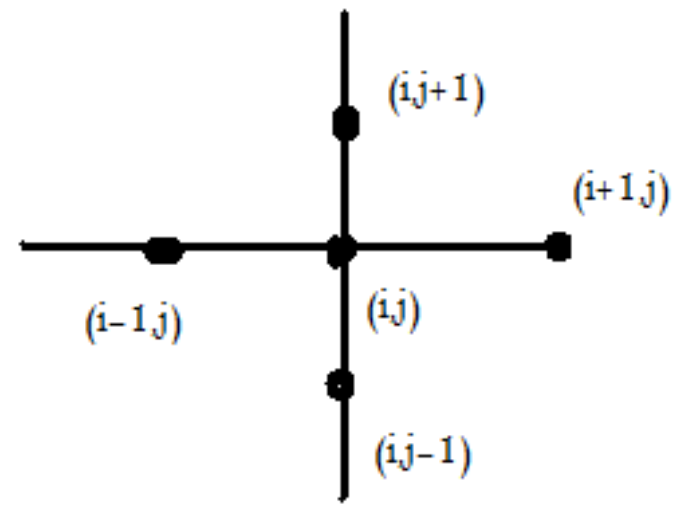

Figure (3.1): Geometry of the five-point star used in 2-D difference equation

Laplace Equation:

Using this notation in Laplace and Poisson equations respectively gives:

$$
\Phi_{i+1}=\frac{1}{4}\left(\Phi_{(i+1, j)}+\Phi_{(i-1, j)}+\Phi_{(i, j+1)}+\Phi_{(i, j-1)}\right.
$$

and Poisson's Equation:

$$
\Phi_{i+1}=\frac{1}{4}\left(\Phi_{(i+1, j)}+\Phi_{(i-1, j)}+\Phi_{(i, j+1)}+\Phi_{(i, j-1)}-\frac{h^{2}}{4} F_{i, j}\right.
$$

So Laplace 's equation in two dimensions may be expressed as:

$$
\nabla^{2} \Phi=\frac{\partial^{2} \Phi}{\partial x^{2}}+\frac{\partial^{2} \Phi}{\partial y^{2}}=\frac{\Phi_{1}+\Phi_{2}+\Phi_{3}+\Phi_{4}-4 \Phi_{0}}{h^{2}}=0
$$

This is known as the five-point star using Fig. (3.1).

Moreover, Poisson's equation in two dimensions may also be expressed as:

$$
\nabla^{2} \Phi=\frac{\partial^{2} \Phi}{\partial x^{2}}+\frac{\partial^{2} \Phi}{\partial y^{2}}=\frac{\Phi_{1}+\Phi_{2}+\Phi_{3}+\Phi_{4}-4 \Phi_{0}}{h^{2}}=F(x, y) .
$$

4- Numerical Example $5 \times 5$ Grid of Voltage Samples

Consider the simple $\mathbf{5} \times \mathbf{5}$ squared region shown in Figure 4 . The electric potential is specified on the conducting boundaries. Our goal is to determine the potential distribution within this region.

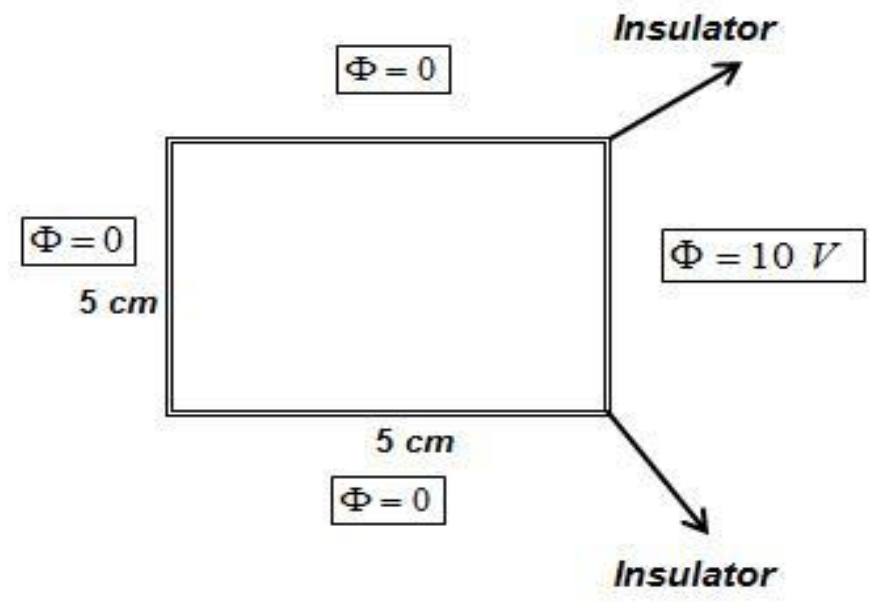

Figure 4a: Geometry of $5 \mathbf{c m} \times 5 \mathbf{c m}$ mesh 
The electric potential everywhere in the squared region should satisfy Laplace's Equation. Using a numerical solutions means we will define $\Phi$ in the squared -

-region of interest by calculating its values at discrete points which are the nodes of a mesh. First of all we must divide the domain in which the potential is to be calculated into a suitable fine grid. So instead of a solution for $\Phi(x, y)$ which provides its continuous variation for a given charge distribution $\rho(x, y)$, the FD solution will provide discrete values of $\Phi$ at the nodes of the established grid. After that we must identify the nodes at which the electric potential is to be calculated. Then we apply the difference equation at each node of the grid to obtain $\mathrm{N}$ equations with $\mathrm{N}$ unknown nodes potentials. The Geometry of $5 \times 5$ mesh is shown in Fig $4 \mathbf{a}$. The value of $\boldsymbol{h}$ (mesh size) in this case is $\boldsymbol{h}=1 \mathrm{~cm}$. The accuracy of the results improves with the reduction in $\boldsymbol{h}$ size.

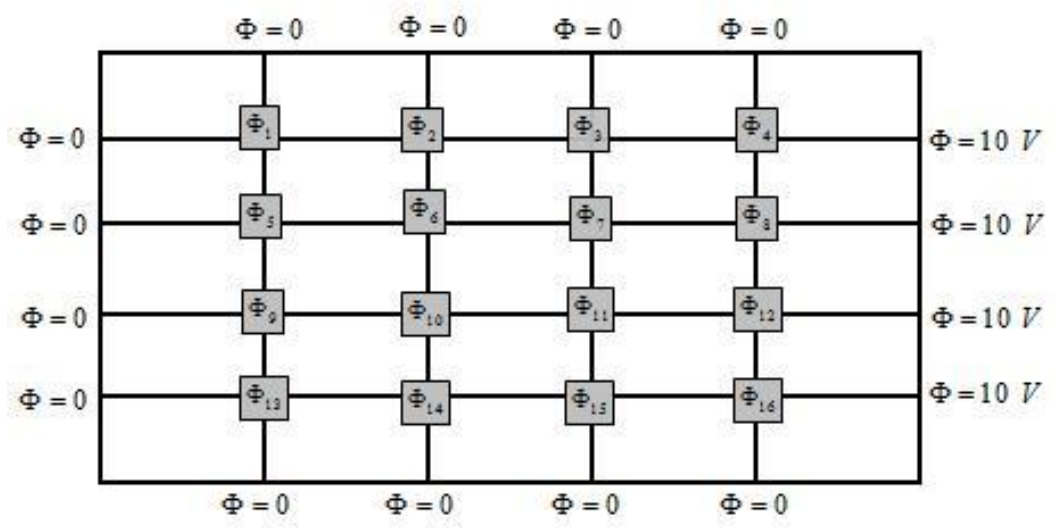

Figure 4b: Sampled grid of voltages

Writing out the full linear system $\mathbf{A} \Phi=\mathrm{b}$, therefore leads to:

\begin{tabular}{|c|c|c|c|c|c|c|c|c|c|c|c|c|c|c|c|c|c|}
\hline-4 & 1 & 0 & 0 & 1 & 0 & 0 & 0 & 0 & 0 & 0 & 0 & 0 & 0 & 0 & 0 & $\Phi_{1}$ & 0 \\
\hline 1 & -4 & 1 & 0 & 0 & 1 & 0 & 0 & 0 & 0 & 0 & 0 & 0 & 0 & 0 & 0 & $\Phi_{2}$ & 0 \\
\hline 0 & 1 & -4 & 1 & 0 & 0 & 1 & 0 & 0 & 0 & 0 & 0 & 0 & 0 & 0 & 0 & $\Phi_{3}$ & 0 \\
\hline 0 & 0 & 1 & -4 & 0 & 0 & 0 & 1 & 0 & 0 & 0 & 0 & 0 & 0 & 0 & 0 & $\Phi_{4}$ & -10 \\
\hline 1 & 0 & 0 & 0 & -4 & 1 & 0 & 0 & 1 & 0 & 0 & 0 & 0 & 0 & 0 & 0 & $\Phi_{5}$ & 0 \\
\hline 0 & 1 & 0 & 0 & 1 & -4 & 1 & 0 & 0 & 1 & 0 & 0 & 0 & 0 & 0 & 0 & $\Phi_{6}$ & 0 \\
\hline 0 & 0 & 1 & 0 & 0 & 1 & -4 & 1 & 0 & 0 & 1 & 0 & 0 & 0 & 0 & 0 & $\Phi_{7}$ & 0 \\
\hline 0 & 0 & 0 & 1 & 0 & 0 & 1 & -4 & 0 & 0 & 0 & 1 & 0 & 0 & 0 & 0 & $\Phi_{8}$ & -10 \\
\hline 0 & 0 & 0 & 0 & 1 & 0 & 0 & 0 & -4 & 1 & 0 & 0 & 1 & 0 & 0 & 0 & $\Phi_{9}$ & 0 \\
\hline 0 & 0 & 0 & 0 & 0 & 1 & 0 & 0 & 1 & -4 & 1 & 0 & 0 & 1 & 0 & 0 & $\Phi_{10}$ & 0 \\
\hline 0 & 0 & 0 & 0 & 0 & 0 & 1 & 0 & 0 & 1 & -4 & 1 & 0 & 0 & 1 & 0 & $\Phi_{11}$ & 0 \\
\hline 0 & 0 & 0 & 0 & 0 & 0 & 0 & 1 & 0 & 0 & 1 & -4 & 0 & 0 & 0 & 1 & $\Phi_{12}$ & -10 \\
\hline 0 & 0 & 0 & 0 & 0 & 0 & 0 & 0 & 1 & 0 & 0 & 0 & -4 & 1 & 0 & 0 & $\Phi_{13}$ & 0 \\
\hline 0 & 0 & 0 & 0 & 0 & 0 & 0 & 0 & 0 & 1 & 0 & 0 & 1 & -4 & 1 & 0 & $\Phi_{14}$ & 0 \\
\hline 0 & 0 & 0 & 0 & 0 & 0 & 0 & 0 & 0 & 0 & 1 & 0 & 0 & 1 & -4 & 1 & $\Phi_{1}$ & 0 \\
\hline 0 & 0 & 0 & 0 & 0 & 0 & 0 & 0 & 0 & 0 & 0 & 1 & 0 & 0 & 1 & -4 & $\Phi$ & -10 \\
\hline
\end{tabular}

Finally, we solve for $\Phi$ either iteratively or by using one of the direct methods to find that

$$
\Phi=\left[\begin{array}{llll}
\Phi_{1} & \Phi_{2} & \Phi_{3} \ldots \Phi_{16}
\end{array}\right]^{T}
$$

The solution for the electric potential at the previous nodes is given as: 


$$
\begin{array}{llll}
\Phi_{1}=0.4545 & \Phi_{2}=1.0985 & \Phi_{3}=2.2348 & \Phi_{4}=4.5455 \\
\Phi_{5}=0.7197 & \Phi_{6}=1.7045 & \Phi_{7}=3.2955 & \Phi_{8}=5.9470 \\
\Phi_{9}=0.7197 & \Phi_{10}=1.7045 & \Phi_{11}=3.2955 & \Phi_{12}=5.9470 \\
\Phi_{13}=0.4545 & \Phi_{14}=1.0985 & \Phi_{15}=2.2348 & \Phi_{16}=4.5455
\end{array}
$$

\section{CONCLUSION}

The finite difference techniques are one of the most popular and efficient methods for solving differential equations. They relate the value of the dependent variable at a point in the solution region to the values at some neighboring points by dividing the solution region into a uniform grid of nodes with a suitable mesh size. Then applying the difference equation at each node of the grid to obtain $\mathrm{N}$ equations with $\mathrm{N}$ unknown node potentials, which can be solved with any numerical methods for solving linear system problems. The accuracy and efficiency of the FD results improves when reducing the mesh size, any reduction in the mesh size results a larger size matrix. Hence a compromise should be made the desired accuracy and the computational time.

\section{REFERENCES}

[1] T. Abassy and H. Zoheiry, Solving Non-Linear Partial Differential Equations Using the Modified Variational Iteration Pade Technique, Journal of Computational and Applied Mathematics, 207: 77-91, 2007.

[2] M. Abdou, Variational Iteration Method for Solving Burger's and Coupled Burger's Equations, J. of Computational and Applied Mathematics, 3: 1-7, 2004.

[3] B. Cockburn, Discontinuous Galerkin Method, Theory, Computational and Applications, Vol.11 of Lecture Notes in Computational Science and Engineering, Springer-Verlag, Berlin.

[4] A. Elmkies and P. Joly, Finite Elements and Mass Lumping for Maxwell's Equations: The 2nd case, Numerical Analysis, C. R. Acad Sci, Paris, 324: 1287-1293, 1997.

[5] L. Elsgolts, Differential Equations and the Calculus of Variations, Translated from the Russian by G. Yankovsky, Mir, Moscow, Applications, 8: 303-309, 1977.

[6] H. Fahs and L. Lanteri, A High-Order Non-Conforming Discontinuous Galerkin Method for Time-Domain Electromagnetic, Journal of Computational and Applied Mathematics, 234: 1088-1096, 2010.

[7] H. Fahs and F. Rapetti, Development of a Non-Conforming Discontinuous Galerkin Method on Simplex Meshes for Electromagnetic Wave Propagation, Fourth International Conference on Advanced Computational Methods in Engineering, 2008.

[8] D. Fleisch, A Stusent's Guide to Maxwell's Equations, $1^{\text {st }}$ edition, Cambridge University Press, 2008.

[9] S. Gedney, Introduction to the Finite Difference Time Domain (FDTD) Method for Electromagnetics (Synthesis Lectures on Computational Electromagnetics), Morgan \& Claypool, 2011.

[10] M. Grote and D. Schotzan, Interior Penalty Discontinuous Galerkin Method for Maxwell's Equations: Energy Norm Error Estimates, Journal of Computational and Applied Mathematics, Volume 204, Issue 2, 15 July 2007, Pages 375386

[11] J.H. He, Variational Iteration Method-Kind of Non-Linear Analytical Technique: Some Examples, International Journal of Non-Linear Mechanics, 34: 699-708, 1997.

[12] J. Hesthaven and T. Warburton, Nodal Discontinuous Galerkin Methods: Algorithm, Analysis and Applications, Springer, 2008.

[13] J. D. Jackson, Classical Electromagnetics, $2^{\text {nd }}$ Ed., Wiley, New York, 1975.

[14] M. Konig and K. Bush, The Discontinuous Galerkin Time-Domain Method for Maxwell's Equations with Anisotropic Materials, Photonics and Nanostructures-Fundamentals, 2010.

[15] S. Momani and S. Abuasad, Application of He's Variational Iteration Method to Helmholtz Equation, Chaos, Solitons and Fractals, 27: 1119-1123, 2005.

[16] P. Monk, Finite Element Method for Maxwell's Equations, Oxford University, NewYourk, 2003.

[17] J. Nagel, Solving the Generalized Poisson Equation Using the Finite Difference Method (FDM), Lecture Notes, Dept. of Electrical and Computer Engineering, University of Utah, 2011.

[18] Mathew. Sadiku, Elements of Electromagnetic, $3^{\text {rd }}$ edition, Oxford Series in Electrical and Computer Engineering 2000.

[19] M. Sadiku, Numerical Techniques in Electromagnetics, $2^{\text {nd }}$ edition, CRC Press, 2000.

[20] N. Sweilam and M. Khader, Convergence of Variational Iteration Method Applied to Non-Linear Coupled System of Partial Differential Equations. Int, J. of Computer Math., 87 (s): 1120-1130, 2010.

[21] N. Sweilam and M. Khader, Variational Iteration Method for One-Dimensional Non-Linear Thermo-Elasticity, Chaos, Solitons and Fractals, 32: 145-149, 2007.

[22] A. Taflove, Computational Electromagnetics: The Finite-Difference Time-Domain Method, Artech House, Boston, 1995.

[23] T. Warburton and J. Hesthaven, Nodal High-Order Nodal Discontinuous Galerkin Methods for Maxwell's Eigenvalue Problem, Philos. Trans. Roy. Soc. London Ser. A, 362:493-524, 2004. 